\title{
2769. Structural state inspection using dual-tree quaternion wavelet transform
}

\author{
Tongqun Ren ${ }^{1}$, Meiling Hui ${ }^{2}$, Xiaodong Wang ${ }^{3}$, Zhiyong Zhang ${ }^{4}$, Junsheng Liang ${ }^{5}$ \\ $1,3,5$ Key Laboratory for Precision and Non-traditional Machining of the Ministry of Education, \\ Dalian University of Technology, Dalian, 116024, China \\ 1, 2, 3, 4,5 Key Laboratory for Micro/Nano Technology and System of Liao Ning Province, \\ Dalian University of Technology, Dalian, 116024, China \\ ${ }^{3}$ Corresponding author

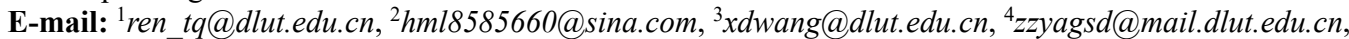 \\ 5jsliang@dlut.edu.cn
}

Received 29 March 2017; received in revised form 30 June 2017; accepted 17 July 2017

DOI https://doi.org/10.21595/jve.2017.18403

Check for updates

\begin{abstract}
The dual-tree quaternion wavelet transform (QWT) was used in conjunction with quaternion-based three-channel joint transmissibility (QTJT) for state inspection. Multiple QTJTs from the same structural state were used to construct a state matrix, which was considered as a feature image. Then QWT coefficients of the feature image were calculated. It supported one magnitude and three phases, in particular, the low-frequency magnitude-phase was set as state feature index. Ultimately, the difference of the state feature indexes were utilized as the state indicator. This method reduced the influence on state inspection caused by measurement uncertainty of single testing sample, because it took overall consideration of multiple testing samples and described the similarity from multiple directions. The availability of suggested method was demonstrated by a real experiment, in which the state changing was realized by loosening fasteners and altering the longitudinal force of rail. This method was also compared with method based on Karhunen-Loeve Transform (K-LT) and artificial neural network (ANN). Experimental result indicated that the suggested method was integrated optimal, moreover, the resolution of the longitudinal force of rail was less than $10 \mathrm{MPa}$ which was equivalent to temperature change of $1.75^{\circ} \mathrm{C}$ for full-lock rail.
\end{abstract}

Keywords: dual-tree quaternion wavelet transform, quaternion-based three-channel joint transmissibility, state inspection, longitudinal stress.

\section{Introduction}

Structural state inspection based on response-only data has recently become a focused research topic, because it does not require a theoretical or numerical model of the measured structure and it is appropriate for structure motivated by unavailable or inaccessible excitation [1-3]. These two advantages are very important for engineering applications.

The key of the response-only method is how to extract feature index being sensitive to small state change. Among the used features, the transmissibility is known as a linear system concept only depended on the intrinsic characteristics of structure. It defines how vibration (both amplitude and phase) is transmitted between two testing points, and provides complete information with respect to the dynamic behavior of the structure. This had given rise to the development of structural state inspection by using transmissibility [4-9]. In fact, the measured vibration value is just the projectional component of the real vibration in sensor's sensitive direction. Hence, the measured transmissibility will deviate from its theoretical value unless the installation orientations of two sensors coincides strictly. Concerning this issue, the QTJT was proposed in our previous work and proved to be unrelated to installation orientation of the sensors, which was advantageous in practical applications [10].

Essentially, the QTJT employed data fusion to avoid only the influence of inconsistent installation orientations of the sensors. More powerful analytical tool should be employed to deal with the measurement uncertainty caused by noise. For example, Yi et al. described the correlation of four-channel signals in quaternion domain, and proposed augmented quaternion singular 
spectrum analysis (AQSSA) based on convex-optimization [11]. Combined convex-optimization and permutation entropy, they also improved tensor-based singular spectrum algorithm (TSSA) to map the vibration signal into a high-dimensional space, which further revealed the dynamic behavior of the original signals [12]. Both two methods were used for fault diagnosis of rolling bearing and obtained good results. Chen et al. trained a four-layer ANN to complete fault diagnosis by using single channel transmissibility [13]. The ANN has strong ability of nonlinear mapping and fault tolerance. It can overcome the input noise to some extent. However, the initial weights of ANN usually need to be adjusted for many times to ensure the network convergence, which results in complicated and time-consuming calculation. And so far, there is no clear rule but an empirical formula to determine the best neural unit number of hidden layer of ANN.

Another way to overcome the measurement uncertainty is to increase the testing sample capacity and utilize the statistical information. For example, Mao and Todd quantified connection stiffness loss by using root-mean-square error directly calculated from the changes of measured transmissibility [14]. The more typical approach is multivariate statistical process monitoring (MSPM). The main idea of MSPM is to decompose the high dimensional training data into low dimensional latent space firstly, and then project the testing data to the latent space to get the monitoring statistic [15-17]. In most MSPM methods, the training data derive from multiple samples but the testing data derive from single sample. To overcome the measurement uncertainty of testing data, it is reasonable to construct testing data based on multiple samples also. Suppose multiple transmissibilities constitute a matrix, which is equivalent to an image in terms of data structure. In this case, structural state inspection converts to problem of image pattern recognition. The QWT was widely used in image pattern recognition due to its representation of quaternion magnitude-phase, which has characteristic of near shift-invariance and the ability to encode phase shifts [18-21].

Based on the above analysis, a state inspection method using dual-tree QWT combined with QTJT was described in this work. The feature image was constructed based on multiple QTJTs from the same state. The magnitude-phase derived from QWT coefficients of the feature image was considered as state feature index. And the difference of the state feature indexes was denoted by the Euclidean distance, which was also utilized as the state indicator. The availability of suggested method was demonstrated by an actual experiment.

\section{Theoretical description}

\subsection{Calculation of QTJT}

Three channel vibration signals were acquired by triple axis accelerometers, and then denoted as time-domain pure quaternion sequence [10]. According to the definition of transmissibility, QTJT between testing point $i, j$ was defined as the ratio of two response frequency spectra of the quaternion sequences, which was calculated as:

$$
Q T_{i j}(\omega)=\frac{A_{Q_{i}}(\omega)}{A_{Q_{j}}(\omega)}=A_{Q_{i}}(\omega) A_{Q_{j}}(\omega)^{-1}=\frac{A_{Q_{i}}(\omega) A_{Q_{j}}(\omega)^{*}}{A_{Q_{j}}(\omega) \cdot A_{Q_{j}}(\omega)^{\prime}},
$$

where $A_{Q}(\omega)$ is the quaternion frequency spectra of vibration signal and $(\bullet)$ indicates dot product of quaternion. Suppose the quaternion vibration signal as a $1 \times N$ color image, $A_{Q}(\omega)$ was calculated based on the quaternion Fourier transform [22, 23]:

$$
A_{Q}(\omega)=\frac{1}{\sqrt{N}} \sum_{t=0}^{N-1} e^{-\mu 2 \pi\left(\frac{t \omega}{N}\right)} a_{q}(t), \quad \mu=\frac{1}{\sqrt{3}}(i+j+k) .
$$


Only brief calculation process was presented here, more details could be found in our previous work [10]. If the structure is one point-driven or motivated by excitation force contained uniform spectral density, the excitation force has no connection with the calculation of QTJT, but only provides exciting energy. This is as the same as transmissibility.

\subsection{Dual-tree QWT}

The dual-tree QWT is a natural extension of discrete wavelet transform (DWT) and complex wavelet transform (CWT). It is constructed based on the 2-D analytic signal and Hilbert transform (HT). Compared with real wavelet transform, QWT contains characteristic of near shift-invariant and provides one amplitude and three phase informations in different scales.

For generalization to 2-D, Bülow introduced a definition of the quaternion analytic signal according to the quaternion Fourier transform (QFT). Given a real 2-D signal $f(x, y)$, its quaternion analytic signal is defined as [24]:

$f^{q}(x, y)=f(x, y)+i f_{H i 1}(x, y)+j f_{H i 2}(x, y)+k f_{H i}(x, y)$,

where $f_{H i 1}(x, y)$ and $f_{H i 2}(x, y)$ are the partial HT along $x$ and $y$ axis, respectively, and $f_{H i}(x, y)$ is the total HT.

The 2-D DWT are formed as tensor products of 1-D DWT along two orthogonal dimensions. The result can be denoted as one scaling function $\varphi(x) \varphi(y)$ and three subband 2-D wavelets $\varphi(x) \psi(y), \psi(x) \varphi(y)$ and $\psi(x) \psi(y)$ that oriented in horizontal, vertical and diagonal directions, respectively [18]. Similarly, the QWT also contains one scaling function and three subband wavelets, which are obtained by constructing the real wavelet and 2-D HTs of a 2-D wavelet as a quaternion according to Eq. (3). Actually, the 2-D HT is just the 1-D HT along either or both axes. To take the diagonal subband real 2-D wavelets $\psi(x) \psi(y)$ as example, its 2-D HT can be calculated as:

$f_{H_{i 1}}\left\{\psi_{h}(x) \psi_{h}(y)\right\}=\psi_{g}(x) \psi_{h}(y)$,

$f_{H_{i 2}}\left\{\psi_{h}(x) \psi_{h}(y)\right\}=\psi_{h}(x) \psi_{g}(y)$,

$f_{H_{i}}\left\{\psi_{h}(x) \psi_{h}(y)\right\}=\psi_{g}(x) \psi_{g}(y)$.

The notion of $\left(\psi_{h}, \psi_{g}\right)$ denotes the wavelet functions corresponding to particular filter $h$ and $\mathrm{g}$, respectively, and they follow the rules: $\left\{\psi_{h}, \psi_{g}=H T\left(\psi_{h}\right)\right\}$. Conveniently, each component in Eq. (4) can be calculated as an alliance of 1-D dual-tree complex wavelets. So, the diagonal subband quaternion wavelet can be given by:

$\psi^{D}(x, y)=\psi_{h}(x) \psi_{h}(y)+i \psi_{g}(x) \psi_{h}(y)+j \psi_{h}(x) \psi_{g}(y)+k \psi_{g}(x) \psi_{g}(y)$.

In the same way, the horizontal and vertical subband quaternion wavelet and the quaternion scaling function can be given by [20]:

$\psi^{H}(x, y)=\varphi_{h}(x) \psi_{h}(y)+i \varphi_{g}(x) \psi_{h}(y)+j \varphi_{h}(x) \psi_{g}(y)+k \varphi_{g}(x) \psi_{g}(y)$,

$\psi^{V}(x, y)=\psi_{h}(x) \varphi_{h}(y)+i \psi_{g}(x) \varphi_{h}(y)+j \psi_{h}(x) \varphi_{g}(y)+k \psi_{g}(x) \varphi_{g}(y)$,

$\varphi(x, y)=\varphi_{h}(x) \varphi_{h}(y)+i \varphi_{g}(x) \varphi_{h}(y)+j \varphi_{h}(x) \varphi_{g}(y)+k \varphi_{g}(x) \varphi_{g}(y)$.

The detailed process of dual-tree QWT can be found in Ref. [21]. The QWT coefficients can be formed as a matrix $F$ as follows: 


$$
F=\left[\begin{array}{llll}
L L_{\varphi_{h}(x) \varphi_{h}(y)} & L H_{\varphi_{h}(x) \psi_{h}(y)} & H L_{\psi_{h}(x) \varphi_{h}(y)} & H H_{\psi_{h}(x) \psi_{h}(y)} \\
L L_{\varphi_{g}(x) \varphi_{h}(y)} & L H_{\varphi_{g}(x) \psi_{h}(y)} & H L_{\psi_{g}(x) \varphi_{h}(y)} & H H_{\psi_{g}}(x) \psi_{h}(y) \\
L L_{\varphi_{h}(x) \varphi_{g}(y)} & L H_{\varphi_{h}}(x) \psi_{g}(y) & H L_{\psi_{h}(x) \varphi_{g}(y)} & H H_{\psi_{h}}(x) \psi_{g}(y) \\
L L_{\varphi_{g}(x) \varphi_{g}(y)} & L H_{\varphi_{g}(x) \psi_{g}(y)} & H L_{\psi_{g}(x) \varphi_{g}(y)} & H H_{\psi_{g}(x) \psi_{g}(y)}
\end{array}\right],
$$

where the first column contains low frequency information, and column 2-4 represents high frequency information in direction of horizontal, vertical and diagonal, respectively. The QWT coefficients provide one magnitude and three phases. The first two phases encode local image shifts, and the third phase carries texture information [21]. Each column of $F$ can constitute a quaternion as follows:

$Q_{L L}=L L_{\varphi_{h}(x) \varphi_{h}(y)}+i L L_{\varphi_{g}(x) \varphi_{h}(y)}+j L L_{\varphi_{h}(x) \varphi_{g}(y)}+k L L_{\varphi_{g}(x) \varphi_{g}(y)}$,
$Q_{L H}=L H_{\varphi_{h}(x) \psi_{h}(y)}+i L H_{\varphi_{g}(x) \psi_{h}(y)}+j L H_{\varphi_{h}(x) \psi_{g}(y)}+k L H_{\varphi_{g}(x) \psi_{g}(y)}$,
$Q_{H L}=H L_{\psi_{h}(x) \varphi_{h}(y)}+i H L_{\psi_{g}(x) \varphi_{h}(y)}+j H L_{\psi_{h}(x) \varphi_{g}(y)}+k H L_{\psi_{g}(x) \varphi_{g}(y)}$,
$Q_{H H}=H H_{\psi_{h}(x) \psi_{h}(y)}+i H H_{\psi_{g}(x) \psi_{h}(y)}+j H H_{\psi_{h}(x) \psi_{g}(y)}+k H H_{\psi_{g}(x) \psi_{g}(y)}$.

To calculate magnitude and phases of the quaternions in Eq. (8) and form a new magnitudephase matrix $A F$ :

$A F=\left|\begin{array}{cccc}\left|Q_{L L}\right| & \varphi_{L L} & \theta_{L L} & \psi_{L L} \\ \left|Q_{L H}\right| & \varphi_{L H} & \theta_{L H} & \psi_{L H} \\ \left|Q_{H L}\right| & \varphi_{H L} & \theta_{H L} & \psi_{H L} \\ \left|Q_{H H}\right| & \varphi_{H H} & \theta_{H H} & \psi_{H H}\end{array}\right|$

where $|\cdot|$ indicates the magnitude and $(\varphi, \theta, \psi)$ indicates the phase angles. Suppose the source image scale is $m \times n$ and the quaternion wavelet decomposition level is $j$, the dimension of $A F$ is $\left(4 m / 2^{j}\right) \times\left(4 n / 2^{j}\right)$.

\subsection{State inspection using QWT and QTJT}

In this work, the modular vectors of multiple QTJTs from the same state were used to construct a state matrix. An equivalent grayscale feature image was obtained via simple scaling transformation of the elements in state matrix. In Fig. 1(a), each row of the image corresponded to a transposed modular vector of one measured QTJT from the same state. In Fig. 1(b), the measured QTJTs were from different states. It can be seen that different samples from the same state contained overall similarity but small local differences caused by measurement uncertainty, while the samples from different states showed obvious distinction.
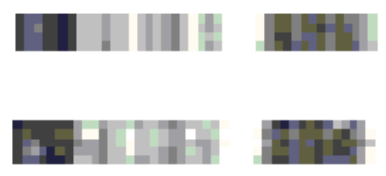

a) The QTJTs from the same state

b) The QTJTs from different states

Fig. 1. The equivalent grayscale image composed of transposed modular vector of measured QTJTs

Before state inspection, the state matrix $V_{s i}^{m \times n}$ for each known state $i$ was constructed firstly, where $m$ was the length of QTJT and $n$ was the amount of QTJTs. Similarly, matrix $V_{t j}^{m \times n}$ for unknown testing state $j$ was also constructed. Then the dual-tree QWT was employed to get the magnitude-phase matrix $A F_{i}$ and $A F_{j}$. Theoretically, if $V_{s i}^{m \times n}$ and $V_{t j}^{m \times n}$ belong to the same state, their corresponding magnitude-phase characteristic embedded in $A F_{i}$ and $A F_{j}$ must be close to 
each other.

The first row of $A F$ indicates the low-frequency magnitude-phase characteristic of original image. The magnitude $\left|Q_{L L}\right|$ takes good low-pass characteristic in both the horizontal and vertical direction, and it is also the approximate representation of the original image in the next scale. Meanwhile, the phase $\left(\varphi_{L L}, \theta_{L L}, \psi_{L L}\right)$ contains the local image shifts and image edge details. Compared with the magnitude-phase characteristic in specific direction, i.e., the last three rows in $A F$, the low-frequency magnitude-phase characteristic contains more complete information of original image. Furthermore, the data size of low-frequency magnitude-phase characteristic is only a quarter of $A F$, which is similar to the data dimension reduction in MSPM. So, the low-frequency magnitude-phase characteristic was selected as the state feature index in this work.

From the above, state inspection can be completed by evaluating the closeness of the state feature index between $A F_{i}$ and $A F_{j}$. Specifically, testing state $j$ can be judged as known state $i$ when their Euclidean distance is the minimal. The flow chart of this method is summarized as Fig. 2. Since the calculation result for each step is uniquely determined, the whole process is clear and unidirectional with no detection or adjustment loop.

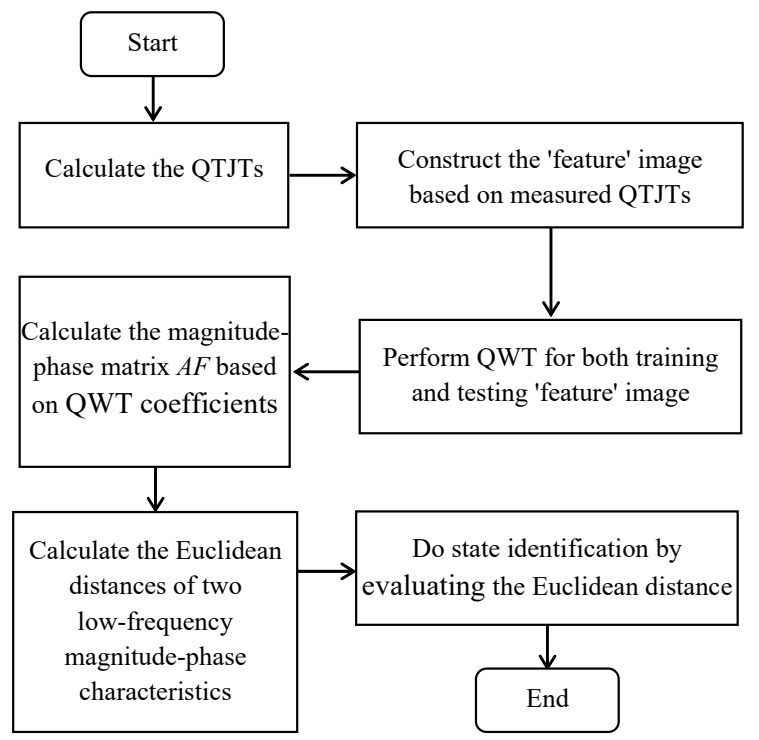

Fig. 2. The flow chart of the QWT-based method

\section{Experiment and discussion}

Based on a real test platform of ballastless track, an experiment was performed. During the experiment, four fasteners between two testing points were loosed in turn. So, five structural states were achieved. The experimental process was just in keeping with that in Ref. [10] except for the motivation times being 40 instead of 20 . The valid frequency was selected from $0.2 \mathrm{kHz}$ to $1.7 \mathrm{kHz}$. Thus, the length of QTJT was 152 because the frequency resolution was $9.76 \mathrm{~Hz}$. All the QTJTs from state 1 was illustrated in Fig. 3, together with the average QTJT from each state. In Fig. 3(a), the legend was not given because there was no enough space for the large amount of QTJTs. Actually, the line type was unified as solid and the color code was cycled every seven QTJTS. Again, the conclusion can be drawn that the QTJTs from the same state coincide approximatively, but the QTJTs from different states disaccord. The relative inconsistency in Fig. 3(a) mainly derived from the measurement uncertainty caused by instrument noise, environmental noise such as passing vehicles around the test platform, tiny changes of the exciting position, etc.

Here, four measured QTJTs from the same state were used to construct a feature image. So, a 
total of 10 feature images with size of $152 \times 4$ were obtained for each state. The former six images were considered as training images from known state and the last four images were used as testing ones from unknown state. Then state inspection was performed according to section 2.3. It was no doubt that higher level of quaternion wavelet decomposition meant higher frequency resolution. But it required more QTJTs to construct big feature image so as to increase calculation cost. Actually, QTJTs from the same state coincided approximatively, so the feature image had no rich textures. Therefore, high decomposition level was not necessary based on overall consideration. Here, the decomposition level was selected as 2. This resulted in $A F$ taking the same size with state matrix. And the size of low-frequency magnitude-phase characteristic was as same as one QTJT. The filter bank used in QWT was designed according to Ref. [25].

All the known feature images were ordered in sequence according to the state No. Thus, the testing feature image from state $i$ should correspond to known images $6 \times(i-1)+1 \sim 6 i$. The inspection result of QWT-based method was summarized in Table 1, where the first column showed the serial number of the matching image from known state, and the second column showed identification results.

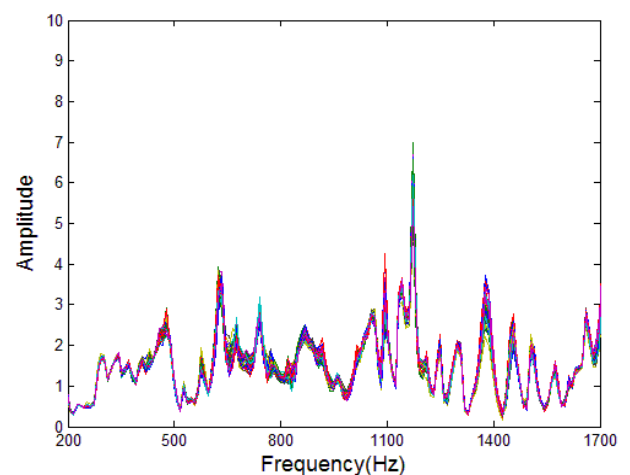

a) All QTJTs from state 1

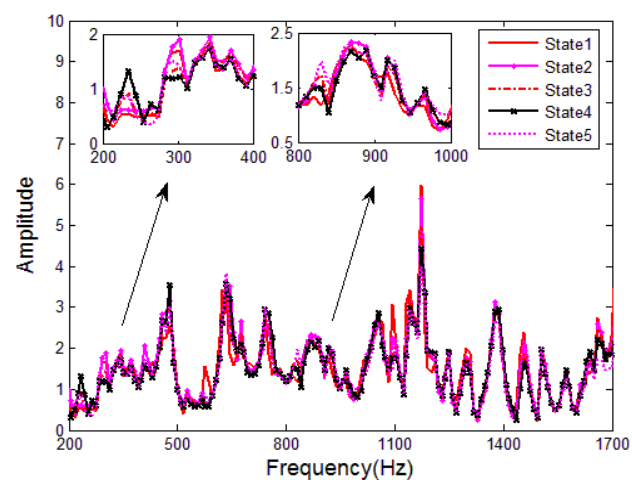

b) Average QTJT of each state

Fig. 3. The measured QTJT

Table 1. State inspection result of QWT-based method

\begin{tabular}{|c|c|c|c|c|c|c|c|c|}
\hline \multirow{2}{*}{ State No. } & \multicolumn{6}{|c|}{ The serial number of matching images and identification results } \\
\cline { 2 - 9 } & Testing image 1 & \multicolumn{2}{|c|}{ Testing image 2 } & \multicolumn{2}{c|}{ Testing image 3 } & Testing image 4 \\
\hline 1 & 5 & $\sqrt{ }$ & 5 & $\sqrt{ }$ & 5 & $\sqrt{ }$ & 5 & $\sqrt{ }$ \\
\hline 2 & 11 & $\sqrt{ }$ & 11 & $\sqrt{ }$ & 11 & $\sqrt{ }$ & 10 & $\sqrt{ }$ \\
\hline 3 & 18 & $\sqrt{ }$ & 18 & $\sqrt{ }$ & 15 & $\sqrt{ }$ & 15 & $\sqrt{ }$ \\
\hline 4 & 24 & $\sqrt{ }$ & 19 & $\sqrt{ }$ & 20 & $\sqrt{ }$ & 23 & $\sqrt{ }$ \\
\hline 5 & 28 & $\sqrt{ }$ & 28 & $\sqrt{ }$ & 28 & $\sqrt{ }$ & 28 & $\sqrt{ }$ \\
\hline
\end{tabular}

In order to test the performance of QWT-based state inspection method, it was compared with K-LT method which was expounded in our previous work [10]. Here, the former 30 QTJTs were training data and the latter 10 QTJTs were testing data. Likewise, all the training QTJTs were ordered in sequence. Thus, given a testing QTJT from state $i$, the serial number of its matching training QTJT should lie in $30 \times(i-1)+1 \sim 30 i$. The inspection result of K-LT method was summarized in Table 2.

According to Table 1 and Table 2, the QWT-based method got $100 \%$ correct inspection rate, but the K-LT method got one misidentification in state 3 . The effect of the two method looks a lot like, but different in deed. If the testing state is state $i$, in theory, any Euclidean distance lied in $6 \times(i-1)+1 \sim 6 i$ should be smaller than other ones when QWT-based method was used, and any Euclidean distance lied in $30 \times(i-1)+1 \sim 30 i$ should be smaller than other ones when K-LT method was used. The Euclidean distances of two arbitrary testing samples from state 3 using above two methods were illustrated in Fig. 4. It was seen that the QWT-based method got 
relative clear and regular result, but the K-LT method got not only one misidentification but also relative confused result even though the minimal Euclidean distance was correct matched. This indicated that the K-LT method might get relative higher chance of misidentification for weak damage.

Table 2. State inspection result of K-LT method

\begin{tabular}{|c|c|c|c|c|c|c|c|c|c|c|}
\hline \multirow{2}{*}{ State No. } & \multicolumn{10}{|c|}{ The serial number of matching training QTJTs and identification results } \\
\hline & \multicolumn{2}{|c|}{ Testing QTJT1 } & \multicolumn{2}{|c|}{ Testing QTJT2 } & \multicolumn{2}{|c|}{ Testing QTJT3 } & \multicolumn{2}{|c|}{ Testing QTJT 4} & \multicolumn{2}{|c|}{ Testing QTJT 5} \\
\hline 1 & 19 & $\sqrt{ }$ & 30 & $\sqrt{ }$ & 22 & $\sqrt{ }$ & 20 & $\sqrt{ }$ & 21 & $\sqrt{ }$ \\
\hline 2 & 31 & $\sqrt{ }$ & 35 & $\sqrt{ }$ & 56 & $\sqrt{ }$ & 40 & $\sqrt{ }$ & 40 & $\sqrt{ }$ \\
\hline 3 & 88 & $\sqrt{ }$ & 88 & $\sqrt{ }$ & 66 & $\sqrt{ }$ & 90 & $\sqrt{ }$ & 72 & $\sqrt{ }$ \\
\hline 4 & 110 & $\sqrt{ }$ & 108 & $\sqrt{ }$ & 109 & $\sqrt{ }$ & 116 & $\sqrt{ }$ & 98 & $\sqrt{ }$ \\
\hline 5 & 135 & $\sqrt{ }$ & 124 & $\sqrt{ }$ & 144 & $\sqrt{ }$ & 146 & $\sqrt{ }$ & 135 & $\sqrt{ }$ \\
\hline State No. & \multicolumn{2}{|c|}{ Testing QTJT6 } & \multicolumn{2}{|c|}{ Testing QTJT7 } & \multicolumn{2}{|c|}{ Testing QTJT8 } & \multicolumn{2}{|c|}{ Testing QTJT9 } & \multicolumn{2}{|c|}{ Testing QTJT10 } \\
\hline 1 & 26 & $\sqrt{ }$ & 17 & $\sqrt{ }$ & 18 & $\sqrt{ }$ & 21 & $\sqrt{ }$ & 18 & $\sqrt{ }$ \\
\hline 2 & 53 & $\sqrt{ }$ & 32 & $\sqrt{ }$ & 57 & $\sqrt{ }$ & 40 & $\sqrt{ }$ & 57 & $\sqrt{ }$ \\
\hline 3 & 90 & $\sqrt{ }$ & 66 & $\sqrt{ }$ & 67 & $\sqrt{ }$ & 90 & $\sqrt{ }$ & 115 & $x$ \\
\hline 4 & 118 & $\sqrt{ }$ & 113 & $\sqrt{ }$ & 116 & $\sqrt{ }$ & 108 & $\sqrt{ }$ & 95 & $\sqrt{ }$ \\
\hline 5 & 148 & $\sqrt{ }$ & 139 & $\sqrt{ }$ & 135 & $\sqrt{ }$ & 150 & $\sqrt{ }$ & 136 & $\sqrt{ }$ \\
\hline
\end{tabular}

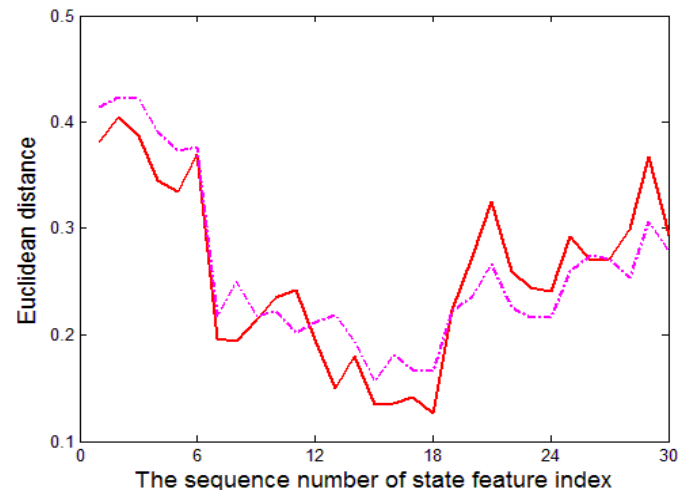

a) The QWT-based method

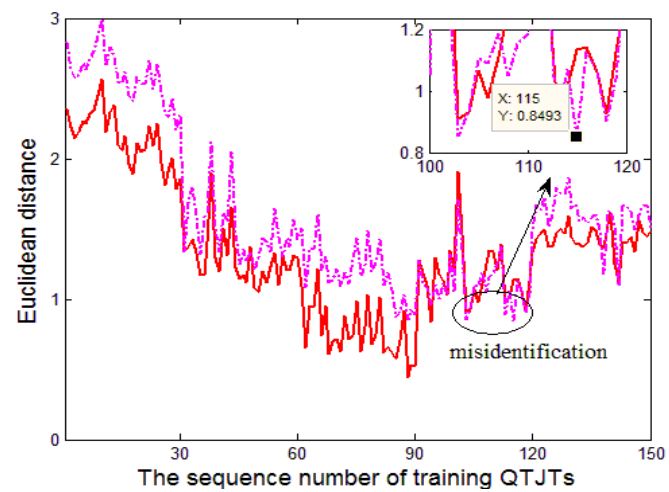

b) The K-LT method

Fig. 4. The Euclidean distances of two arbitrary testing samples from state 2

The QWT-based method was also compared with ANN method. Based on MATLAB, a threelayer BP ANN was created and trained. The normalized modular vector of the measured QTJT was utilized as the input, and the corresponding expected output was a $n$-dimensional vector, where $n$ was the amount of known state. If the input belonged to state $i$, the $i$ th element of the expected output vector was 1 but others were 0 . The learning rate of ANN was 0.05, the error threshold of objective function was $10^{-3}$ and the maximum iterations were 1000 . For each state, the former 20 measured QTJTs were utilized as training QTJTs, the latter 10 measured QTJTs were utilized as verification QTJTs to adjust the ANN structure, and the latest 10 measured QTJTs were utilized as testing QTJTs. For each testing QTJT, we calculated the Euclidean distances between its actual output vector and the expected output vectors of all the known state, and then judged the testing state was just one specific known state when their ANN outputs matched up to each other.

In this work, we premised the difference of corresponding elements of matching vectors should be no more than 0.3. So, the matching vectors must get the minimal Euclidean distance which was less than $0.3 \sqrt{5} \approx 0.67$ at the same time due to five experimental states. Given a set of random initial values of the network weights, the ANN converged to the pre-set precision and got the structure of 152-12-5 after 12 iterations. The Euclidean distance results for all the five experimental states were illustrated in Fig. 5(a)-5(e). 
2769. STRUCTURAL STATE INSPECTION USING DUAL-TREE QUATERNION WAVELET TRANSFORM.

TongQun Ren, Meiling Hui, XiaOdong Wang, Zhiyong Zhang, Junsheng Liang

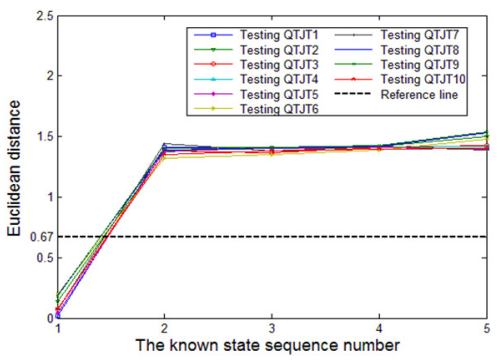

a)

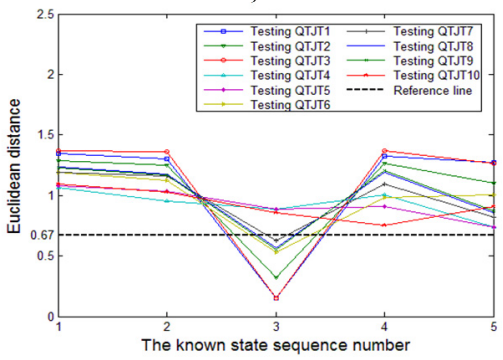

c)

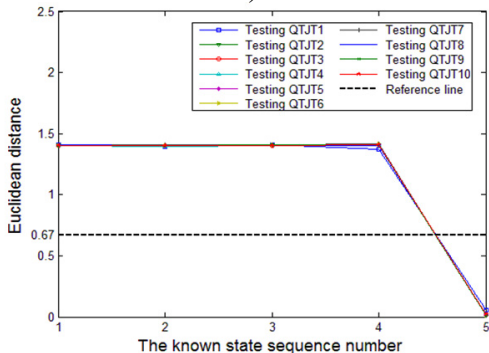

e)

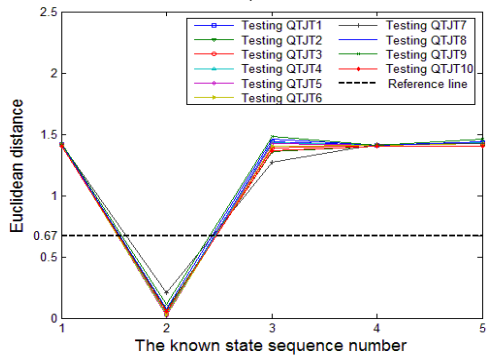

g)

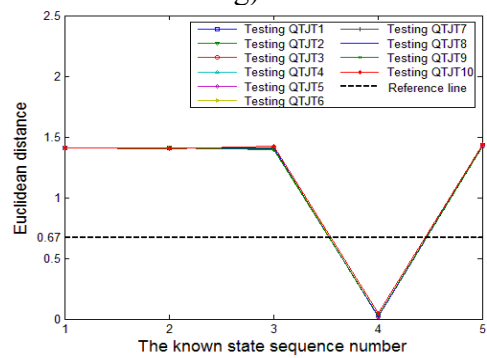

i)

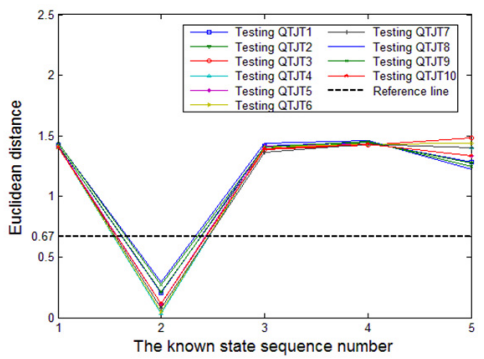

b)

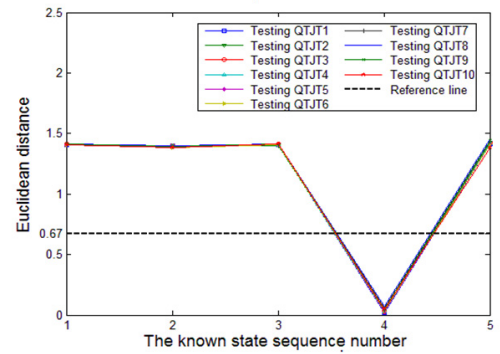

d)

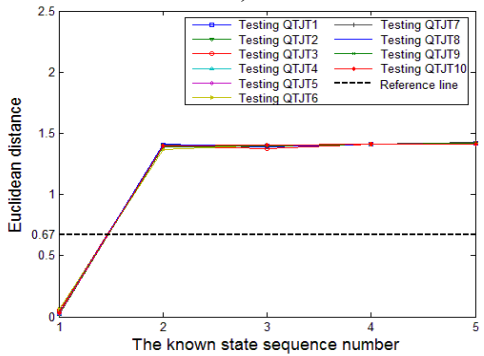

f)

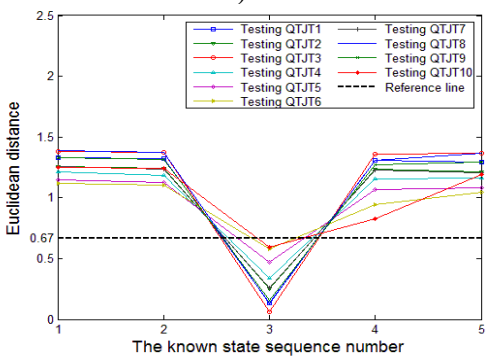

h)

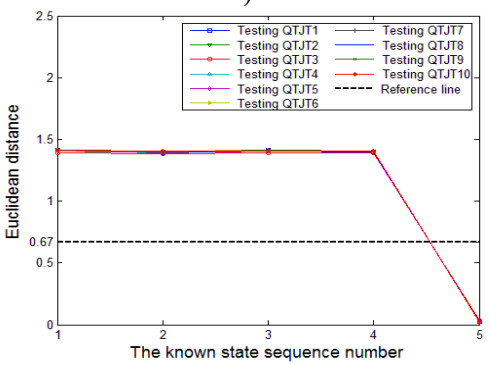

j)

Fig. 5. The Euclidean distances between actual output of the testing QTJTs and the expected output vectors of all the known states. a)-e) testing QTJTs from state 1-5 with ANN structure of 152-12-5

f)-j) testing QTJTs from state 1-5 with ANN structure of 152-14-5 
As shown in Fig. 5(c), the testing QTJT4, QTJT 5 and QTJT 10 of state 3 were misidentified. Moreover, the misidentifications of both ANN and K-LT method were focused on state 3 simultaneously.

As mentioned above, there is no clear rule but an empirical formula to determine the best neural unit number of hidden layer of ANN. Usually, the training accuracy of ANN can be improved by increasing the neural unit number of hidden layer, but in the meantime, the training efficiency may be reduced due to complex network structure. Here, the ANN was retrained with structure of 152-14-5. Given a set of new random initial values of the network weights, the ANN converged to the pre-set precision after 8 iterations, and then the state inspection was redone with the new ANN. The Euclidean distance results for all the five experimental states were illustrated in Fig. 5(f)-5(j). In this case, all the testing QTJTs were identified correctly, but the relatively worse Euclidean distance results were still obtained in state 3, as shown in Fig. 5(h). This indicated that the measured QTJTs from sate 3 were polluted more seriously than others. Note that the cutoff value was just a user-defined value by experience. Here the cutoff value less than 0.3 will lead to worse identification results according to Fig. 5(c) and Fig. 5(h).

Note that both two ANNs with different structure met the pre-set precision but got different inspection result in this work. Moreover, the ANN structure and the initial values of network weights affected the training efficiency. These were the issues that required attention when ANN method was employed. In a word, the QWT-based method got similar inspection effect but clearer and simpler computation process than ANN method, as well as better performance to overcome the measurement uncertainty than K-LT method.

Luo et al. had proved that the longitudinal stress of the rail affected the vibration characteristics of the continuous welded rail track structure [26]. In order to verify this idea, a new experiment was performed by using a LG-900 hydraulic rail tensor to change the longitudinal stress of the rail, as shown in Fig. 6. Before the longitudinal stress changed, all the fasteners of the whole track structure were loosened. The hydraulic rail tensor was utilized to provide a new stress. Then torque spanners were used to re-lock all the fasteners so as to ensure the same locking force of the fasteners before and after longitudinal stress changes.

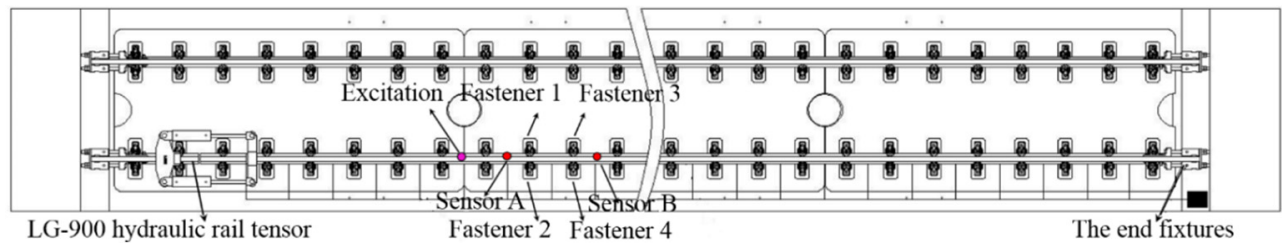

a) The schematic diagram of experimental scene

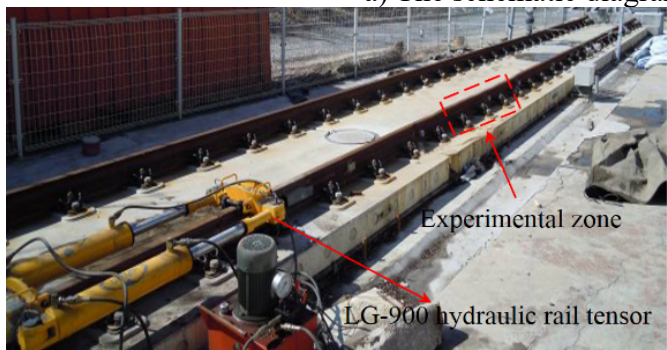

b) The actual test platform of ballastless track

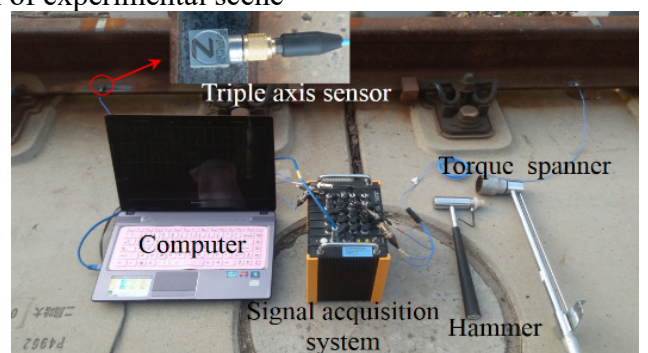

c) The used experimental equipment

Fig. 6. The experimental scene and equipment

Given a specific longitudinal stress, the four fasteners between the same two testing points were also loosened one after another. Here, six tension stresses were provided, i.e., $0 \mathrm{MPa}$, $10 \mathrm{MPa}, 20 \mathrm{MPa}, 30 \mathrm{MPa}, 40 \mathrm{MPa}$ and $50 \mathrm{MPa}$. For each locking condition at one specific stress, 20 measured QTJTs were acquired. For each longitudinal stress, the average of all the 20 measured QTJTs belonged to the same locking condition was calculated. The average QTJT of the first two 
locking condition was illustrated in Fig. 7. It was seen that there were obvious differences among the average QTJT from the same lock condition but different longitudinal stress. Therefore, the structural state should be thought to have changed when the longitudinal stress changed to a certain degree even though any else condition remained the same. In this work, we considered that a total of 30 states were obtained. The state number was ordered following the rules in Table 3.

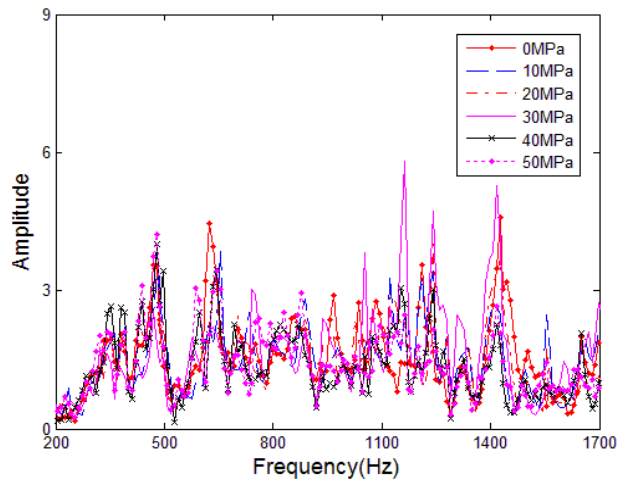

a) All fasteners fastened

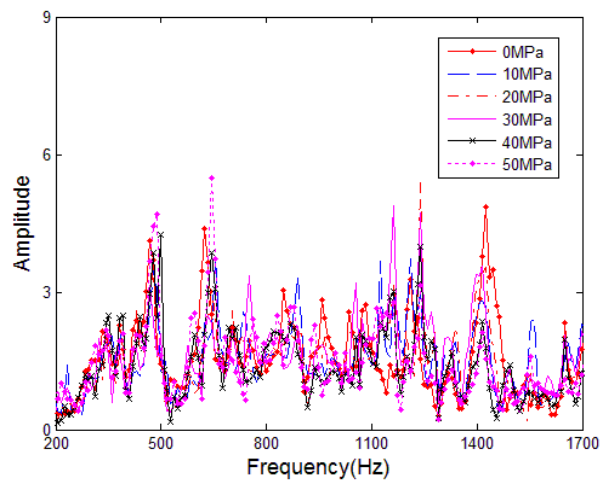

b) One fastener loosened

Fig. 7. The average QTJT from different longitudinal stress

Table 3. The order rule of structural state number

\begin{tabular}{|c|c|c|c|c|c|c|c|c|c|}
\hline Locking condition & \multicolumn{3}{|c|}{$\begin{array}{c}1 \\
\text { All fasteners fastened } \\
\end{array}$} & \multicolumn{3}{|c|}{$\begin{array}{c}2 \\
\text { One fastener loosened }\end{array}$} & \multicolumn{3}{|c|}{$\begin{array}{c}5 \\
\text { All fasteners loosened }\end{array}$} \\
\hline Longitudinal stress & $0 \mathrm{MPa}$ & & $50 \mathrm{MPa}$ & $0 \mathrm{MPa}$ & & $50 \mathrm{MPa}$ & $0 \mathrm{MPa}$ & & $50 \mathrm{MPa}$ \\
\hline State No. & State 1 & & State 6 & State 7 & & State 12 & State 25 & & State 30 \\
\hline
\end{tabular}

Based on the new experimental data, state inspection using QWT-based method was performed firstly. A total of 5 feature images with size of $152 \times 4$ were obtained for each state. The former three images were considered as training images from known state and the latter two images were used as testing ones from unknown state. According to Table 3, all the known feature images were ordered in sequence. Thus, the testing images from state $i$ should correspond to known images $3 \times(i-1)+1 \sim 3 i$. The state inspection result was summarized in Table 4 , in which $100 \%$ correct identification rate was obtained again.

In order to present the inspection result more intuitively, the Euclidean distances of the two testing samples in locking condition 2 at each longitudinal stress were illustrated in Fig. 8.

Table 4. State inspection result of QWT-based method

\begin{tabular}{|c|c|c|c|c|c|c|c|c|c|c|c|c|}
\hline \multirow[b]{2}{*}{$\begin{array}{l}\text { Locking } \\
\text { condition }\end{array}$} & \multicolumn{12}{|c|}{ The serial number of matching feature images and identification results } \\
\hline & \multicolumn{2}{|c|}{$\begin{array}{c}\text { Testing image } \\
1 \text { at } 0 \mathrm{MPa}\end{array}$} & \multicolumn{2}{|c|}{$\begin{array}{c}\text { Testing image } \\
2 \text { at } 0 \mathrm{MPa}\end{array}$} & \multicolumn{2}{|c|}{$\begin{array}{c}\text { Testing image } \\
1 \text { at } 10 \mathrm{MPa}\end{array}$} & \multicolumn{2}{|c|}{$\begin{array}{l}\text { Testing image } \\
2 \text { at } 10 \mathrm{MPa}\end{array}$} & \multicolumn{2}{|c|}{$\begin{array}{l}\text { Testing image } \\
1 \text { at } 20 \mathrm{MPa}\end{array}$} & \multicolumn{2}{|c|}{$\begin{array}{l}\text { Testing image } \\
2 \text { at } 20 \mathrm{MPa}\end{array}$} \\
\hline 1 & 3 & $\sqrt{ }$ & 1 & $\sqrt{ }$ & 5 & $\sqrt{ }$ & 6 & $\sqrt{ }$ & 9 & $\sqrt{ }$ & 7 & $\sqrt{ }$ \\
\hline 2 & 21 & $\sqrt{ }$ & 20 & $\sqrt{ }$ & 23 & $\sqrt{ }$ & 24 & $\sqrt{ }$ & 25 & $\sqrt{ }$ & 26 & $\sqrt{ }$ \\
\hline 3 & 39 & $\sqrt{1}$ & 39 & $\sqrt{ }$ & 40 & $\sqrt{ }$ & 41 & $\sqrt{ }$ & 43 & $\sqrt{ }$ & 44 & $\sqrt{1}$ \\
\hline 4 & 57 & $\sqrt{ }$ & 57 & $\sqrt{ }$ & 60 & $\sqrt{ }$ & 58 & $\sqrt{ }$ & 63 & $\sqrt{ }$ & 63 & $\sqrt{ }$ \\
\hline 5 & 74 & $\sqrt{ }$ & 75 & $\sqrt{ }$ & 76 & $\sqrt{ }$ & 77 & $\sqrt{ }$ & 81 & $\sqrt{ }$ & 79 & $\sqrt{ }$ \\
\hline $\begin{array}{l}\text { Locking } \\
\text { condition }\end{array}$ & \multicolumn{2}{|c|}{$\begin{array}{l}\text { Testing image } \\
1 \text { at } 30 \mathrm{MPa}\end{array}$} & \multicolumn{2}{|c|}{$\begin{array}{c}\text { Testing image } \\
2 \text { at } 30 \mathrm{MPa}\end{array}$} & \multicolumn{2}{|c|}{$\begin{array}{l}\text { Testing image } \\
1 \text { at } 40 \mathrm{MPa}\end{array}$} & \multicolumn{2}{|c|}{$\begin{array}{l}\text { Testing image } \\
2 \text { at } 40 \mathrm{MPa}\end{array}$} & \multicolumn{2}{|c|}{$\begin{array}{c}\text { Testing image } \\
1 \text { at } 50 \mathrm{MPa}\end{array}$} & \multicolumn{2}{|c|}{$\begin{array}{l}\text { Testing image } \\
2 \text { at } 50 \mathrm{MPa}\end{array}$} \\
\hline 1 & 10 & $\sqrt{ }$ & 10 & $\sqrt{ }$ & 14 & $\sqrt{ }$ & 13 & $\sqrt{ }$ & 18 & $\sqrt{ }$ & 18 & $\sqrt{ }$ \\
\hline 2 & 29 & $\sqrt{ }$ & 28 & $\sqrt{ }$ & 33 & $\sqrt{ }$ & 33 & $\sqrt{ }$ & 36 & $\sqrt{ }$ & 34 & $\sqrt{ }$ \\
\hline 3 & 48 & $\sqrt{ }$ & 47 & $\sqrt{ }$ & 49 & $\sqrt{ }$ & 51 & $\sqrt{ }$ & 53 & $\sqrt{ }$ & 53 & $\sqrt{ }$ \\
\hline 4 & 65 & $\sqrt{ }$ & 65 & $\sqrt{ }$ & 68 & $\sqrt{ }$ & 68 & $\sqrt{ }$ & 71 & $\sqrt{ }$ & 72 & $\sqrt{ }$ \\
\hline 5 & 83 & $\sqrt{ }$ & 83 & $\sqrt{ }$ & 87 & $\sqrt{ }$ & 87 & $\sqrt{ }$ & 90 & $\sqrt{ }$ & 88 & $\sqrt{ }$ \\
\hline
\end{tabular}




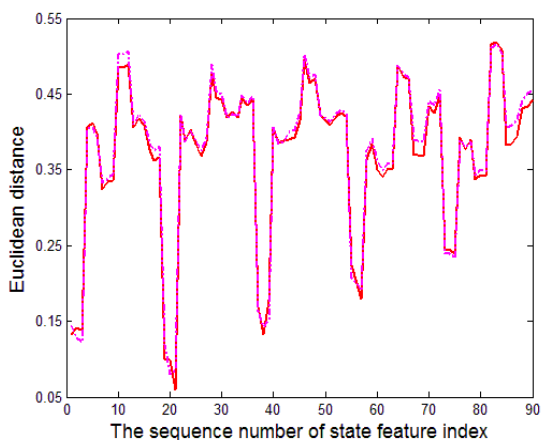

a) At $0 \mathrm{MPa}$

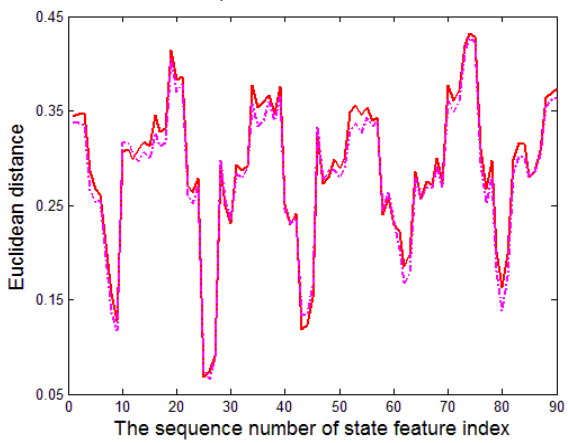

c) At $20 \mathrm{MPa}$

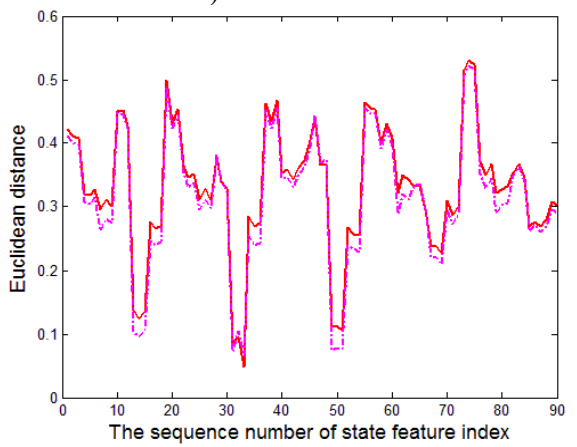

e) At $40 \mathrm{MPa}$

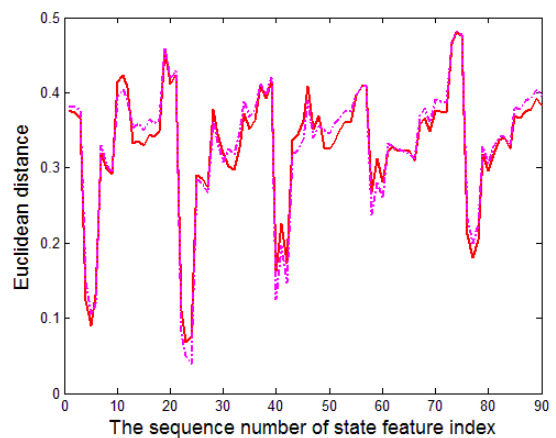

b) At $10 \mathrm{MPa}$

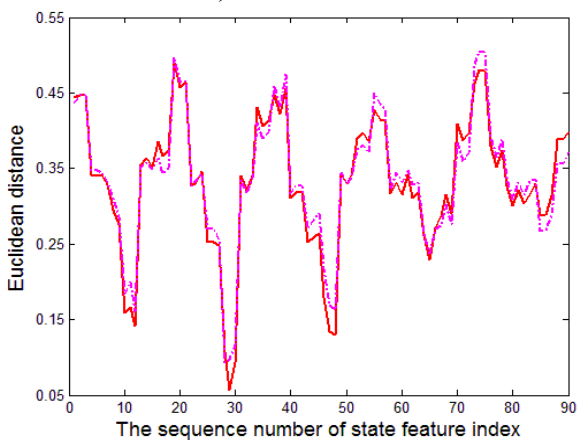

d) At $30 \mathrm{MPa}$

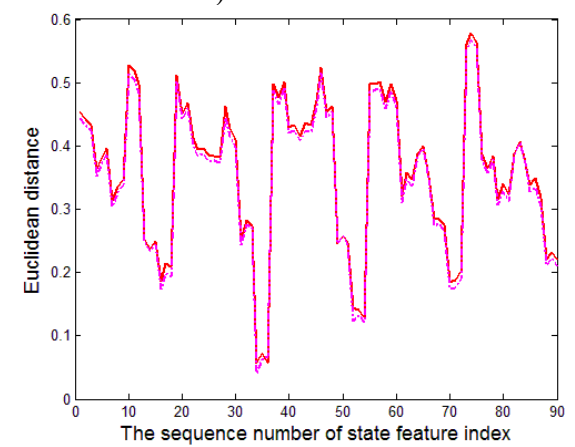

f) At $50 \mathrm{MPa}$

Fig. 8. The Euclidean distances of two testing samples in locking condition 2

The experimental result indicated that the resolution of QWT-based method for longitudinal stress change of the rail was superior to $10 \mathrm{MPa}$. According to the railway rail standards, the coefficient of linear expansion of the rail is $\alpha=0.0118 \mathrm{~mm} /\left(\mathrm{m} \cdot{ }^{\circ} \mathrm{C}\right)$, the elasticity modulus of the rail is $E=2.1 \times 10^{5} \mathrm{MPa}$, and the cross-sectional area of the rail is $7.745 \times 10^{-3} \mathrm{~m}^{2}$. When the rail is completely locked, i.e., no longitudinal extension was permitted, the longitudinal force of the rail caused by temperature change of $1{ }^{\circ} \mathrm{C}$ can be given by:

$$
\begin{aligned}
F & =\sigma A=E \frac{\Delta L}{L} A=E \cdot \alpha \cdot T \cdot A \\
& =2.1 \times 10^{5} \mathrm{MPa} \times 11.8 \times 10^{-6} \times 7.745 \times 10^{-3} \mathrm{~m}^{2}=19192.11 \mathrm{~N} .
\end{aligned}
$$

The cross-sectional area of the oil cylinder of LG-900 hydraulic rail tensor was $3.352 \times 10^{-3} \mathrm{~m}^{2}$. So, the longitudinal force corresponding to stress change of $10 \mathrm{MPa}$ could be calculated as $10 \mathrm{MPa} \times 3.352 \times 10^{-3} \mathrm{~m}^{2}=33520 \mathrm{~N}$. Thus, the equivalent resolution of QWT-based method for 
temperature change was superior to $33520 \mathrm{~N} / 19192.11 \mathrm{~N} \times 1{ }^{\circ} \mathrm{C}=1.75^{\circ} \mathrm{C}$. For the reason of large experimental workload, smaller longitudinal stress change was not performed.

Subsequently, the new experimental data was also handled by K-LT method. This time, one misidentification still occurred in state 10 , moreover, the obviously confused results were obtained in state 11. Part of inspection result of K-LT method was summarized in Fig. 9, where only six training QTJTs and two arbitrary testing QTJTs were illustrated in order to evaluate the regularity of the detection result compared with Fig. 4(a).

Frankly speaking, both the QWT-based method and K-LT method looked "ideal" in this work. The "ideal" classification result indicated that the QTJT was sensitive to full loose of one fastener in spite of the longitudinal stress, which could be considered as small change of the track structure. Actually, the ANN method was also employed at last. The three-layer BP ANN failed to converge to the pre-set precision after several attempts. In this work, no complex network structures were tried.

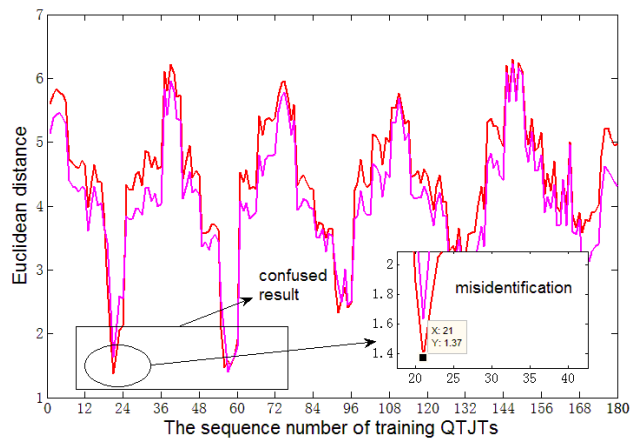

a) QTJTs from state 10

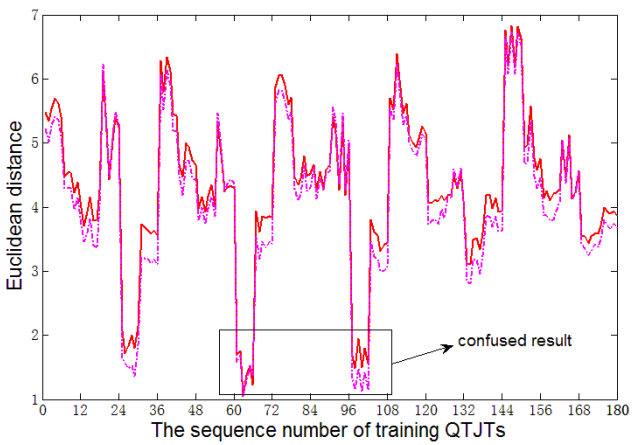

b) QTJTs from state 11

Fig. 9. The Euclidean distances of two arbitrary testing samples using K-LT method

Meiling Hui contributes significantly to the computational process. Xiaodong Wang contributes significantly to the experimental design and principle analysis of QWT. Zhiyong Zhang implement the experiment and complete basic data processing. Junsheng Liang participates in the experiment and improves the writing of English.

\section{Conclusions}

Learning from technology of image pattern recognition and MSPM, state inspection method using dual-tree QWT combined with QTJT was described. Multiple measured QTJTs were used to form a state feature image. Then the Euclidean distance between low-frequency magnitude-phase characteristics of different state feature images were utilized as the state indicator. The suggested method inspected the similarity of two feature images based on both magnitude and phase that contained the local image shifts and edge details. Moreover, it also reduced the influence of measurement uncertainty of single measured QTJT because it took overall consideration of multiple measured QTJTs. It was compared with K-LT and ANN method through a real experiment, and proved to be the integrated optimal method that got clearer and simpler computation process than ANN method, as well as better performance to overcome the measurement uncertainty than K-LT method. In addition, it was also proved that the longitudinal stress of object structure should be taken into consideration during state inspection based on vibration characteristic such as QTJT (transmissibility). For the suggested method, the resolution of the longitudinal force of rail was superior to $10 \mathrm{MPa}$ which is equivalent to temperature change of $1.75^{\circ} \mathrm{C}$ for a completely locked railway structure.

This work still emphasized on the extraction of the state feature index and the definition of the state indicator instead of classification method. The suggested method only identified the presence 
of state changes. Therefore, further research should be done to develop it to identify the level and location of state changes. Compared to other method, it needed more measured QTJTs. Actually, this was not a real problem with modern technology of signal acquisition and storage.

\section{Acknowledgements}

This work was supported by the National Natural Science Foundation of China (No. 51305064), the Fundamental Research Funds for the Central Universities (No. DUT16TD20), and the Science Fund for Creative Research Groups of NSFC (51621064).

\section{References}

[1] Yan Y. J., Cheng L., Wu Z. Y., Yam L. H. Development in vibration-based structural damage detection technique. Mechanical Systems and Signal Processing, Vol. 21, Issue 5, 2007, p. 2198-2211.

[2] Zhong S. C., Oyadiji S. O., Ding K. Response-only method for damage detection of beam-like structures using high accuracy frequencies with auxiliary mass spatial probing. Journal of Sound and Vibration, Vol. 311, Issues 3-5, 2008, p. 1075-1099.

[3] Fan W., Qiao P. Z. Vibration-based damage identification methods: a review and comparative study. Structural Health Monitoring, Vol. 10, Issue 1, 2011, p. 83-111.

[4] Feng L., Yi X. H., Zhu D. P., Xie X. Y., Wang Y. Damage detection of metro tunnel structure through transmissibility function and cross correlation analysis using local excitation and measurement. Mechanical Systems and Signal Processing, Vol. 60, Issue 61, 2015, p. 59-74.

[5] Maia Nuno M. M., Almeida Raquel A. B., Urgueira António P. V., Sampaio Rui P. C. Damage detection and quantification using transmissibility. Mechanical Systems and Signal Processing, Vol. 25, Issue 7, 2011, p. 2475-2483.

[6] Johnson T. J. Analysis of Dynamic Transmissibility as a Feature for Structural Damage Detection. Ph.D. Dissertation, Purdue University, USA, 2002.

[7] Lang Z. Q., Park G., Farrar C. R., Todd M. D., Mao Z., Zhao L., Worden K. Transmissibility of non-linear output frequency response functions with application in detection and location of damage in MDOF structural systems. International Journal of Non-Linear Mechanics, Vol. 46, Issue 6, 2011, p. 841-853.

[8] Li J., Hao H., Xia Y., Zhu H. P. Damage detection of shear connectors in bridge structures with transmissibility in frequency domain. International Journal of Structural Stability and Dynamics, Vol. 14, Issue 2, 2014, https://doi.org/10.1142/S0219455413500612.

[9] Johnson T. J., Adams D. E. Transmissibility as a differential indicator of structural damage. Journal of Vibration and Acoustics, Vol. 124, Issue 4, 2002, p. 634-641.

[10] Ren T. Q., He L., Wang D. Z., Liang J. S., Hui M. L. Structural state detection using quaternionbased three-channel joint transmissibility. Journal of Vibroengineering, Vol. 17, Issue 2, 2015, p. 928-938.

[11] Yi C., Lv Y., Dang Z., Xiao H., Yu X. Quaternion singular spectrum analysis using convex optimization and its application to fault diagnosis of rolling bearing. Measurement, Vol. 103, 2017, p. 321-332.

[12] Yi C., Lv Y., Ge M., Xiao H., Yu X. Tensor singular spectrum decomposition algorithm based on permutation entropy for rolling bearing fault diagnosis. Entropy, Vol. 19, 4, p. 2017-139.

[13] Chen Q., Chan Y. W., Worden K. Structural fault diagnosis and isolation using neural networks based on response-only data. Computers and Structures, Vol. 81, Issues 22-23, 2003, p. 2165-2172.

[14] Mao Z., Todd M. A structural transmissibility measurements-based approach for system damage detection. Proceedings of SPIE Conference on Health Monitoring of Structural and Biological Systems, San Diego, USA, 2010.

[15] Garcia Alvarez D., Fuente M. J., Sainz G. I. Fault detection and isolation in transient states using principal component analysis. Journal of Process Control, Vol. 22, Issue 3, 2012, p. 551-563.

[16] Zhou D. H., Li G., Qin S. J. Total projection to latent structures for process monitoring. AIChE Journal, Vol. 56, Issue 1, 2010, p. 168-178.

[17] Matej Ž., Samo Z., Ivan P. EEMD-based multiscale ICA method for slewing bearing fault detection and diagnosis. Journal of Sound and Vibration, Vol. 370, 2016, p. 394-423.

[18] Chan W. L., Choi H., Baraniuk R. G. Quaternion wavelets for image analysis and processing. International Conference on Image Processing, Singapore, Vol. 2, 2004, p. 3057-3060. 
[19] Kadir M., Djebbouri M., Carré P. Magnitude-phase of the dual-tree quaternionic wavelet transform for multispectral satellite image denoising. EURASIP Journal on Image and Video Processing, Vol. 1, 2014, p. 1-16.

[20] Gai S., Luo L. M. Visual objects tracking and identification based on reduced quaternion wavelet transform. Signal, Image and Video Processing, Vol. 8, Issue 1, 2014, p. 75-84.

[21] Chan W. L., Choi H., Baraniuk R. G. Coherent multiscale image processing using quaternion wavelets. IEEE Transactions on Image Processing, Vol. 17, Issue 7, 2008, p. 1069-1082.

[22] Ell T. A. Quaternion-Fourier transforms for analysis of two-dimensional linear time-invariant partialdifferential systems. Proceedings of IEEE Conference Decision and Control, San Antonio, TX, USA, Vol. 1, Issue 4, 1993, p. 1830-1841.

[23] EIl T. A., Sangwine S. J. Hypercomplex Fourier transforms of color images. IEEE Transactions on Image Processing, Vol. 16, 2007, p. 22-35.

[24] Bülow T., Sommer G. A novel approach to the 2D analytic signal. 8th International Conference on Computer Analysis of Images and Patterns, Ljubljana, Slovenia, 1999, p. 25-32.

[25] Kingsbury N. A dual-tree complex wavelet transform with improved orthogonality and symmetry properties. IEEE International Conference on Image Processing, Vancouver, Canada, Vol. 2, 2000, p. 375-378.

[26] Luo Y., Li L., Yin H. A Dynamic analysis of a continuous welded rail track under a longitudinal stress caused by temperature changes. Proceedings of the Institution of Mechanical Engineers, Part F: Journal of Rail and Rapid Transit, Vol. 224, Issue 2, 2010, p. 91-101.

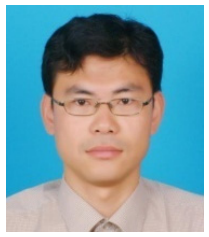

Tongqun Ren received Ph.D. degree in precision measuring technology and instruments from Tianjin University, Tianjin, China, in 2008. He is now an Associate Professor in School of Mechanical Engineering, Dalian University of Technology, China. His research interests include vibration detection, signal analysis and computer vision.

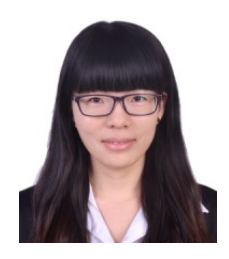

Meiling Hui received Master degree from School of Mechanical Engineering, Dalian University of Technology, Dalian, China. Her current research interests include fault diagnosis and signal analysis.

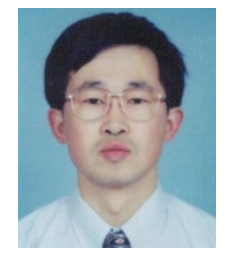

Xiaodong Wang received Ph.D. degree in mechanical and electrical control and automation from Harbin Institute of Technology, Harbin, China, in 1995. He is now an Professor in School of Mechanical Engineering, Dalian University of Technology, Dalian, China. His research interests include signal analysis and micro assembly technology.

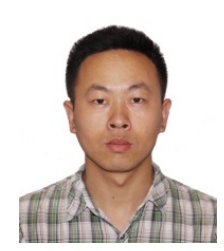

Zhiyong Zhang received Master degree from School of Mechanical Engineering, Dalian University of Technology, Dalian, China. He is now a Ph.D. candidate and his current research interests include fault diagnosis and signal analysis.

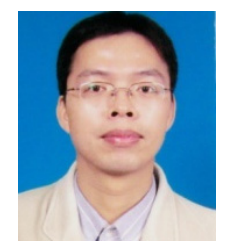

Junsheng Liang received Ph.D. degree in mechanical and electronic engineering from Dalian University of Technology, China, in 2007. He is now an Professor in School of Mechanical Engineering, Dalian University of Technology, China. His research interests include technology of measurement and control, structural health monitoring. 